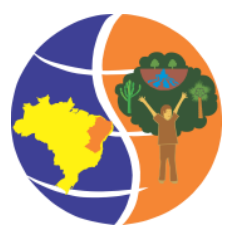

II Simpósio Brasileiro de Recursos Naturais do Semiárido - SBRNS

"Convivência com o Semiárido: Certezas e Incertezas"

Quixadá - Ceará, Brasil

27 a 29 de maio de 2015

doi: 10.18068/IISBRNS2015.resap299

ISSN: 2359-2028

\title{
DETERMINAÇÃO DO BALANÇO DE ENERGIA COM IMAGENS LANDSAT 8 NO PERÍMETRO DE IRRIGAÇÃO NILO COELHO
}

\author{
Antônio Heriberto de Castro Teixeira ${ }^{1}$ \\ ${ }^{1}$ Ph.D., Embrapa Monitoramento por Satélite, Fone: (19) 3211-6200, e-mail: heriberto.teixeira@embrapa.br
}

RESUMO: No perímetro de irrigação Nilo Coelho, situado na região semiárida do Brasil, a vegetação natural, Caatinga, vem sido substituída pela agricultura irrigada, trazendo importância da quantificação dos componentes do balanço de energia em larga escala. Três imagens do satélite Landsat 8 e quatro estações agrometeorológicas foram usadas em conjunto durante períodos sob diferentes condições termo hidrológicas do ano de 2014, para a modelagem destes componentes nestes ecossistemas mistos. Nas culturas irrigadas, o saldo de radiação $(\mathrm{Rn})$ médio variou de 9,1 a $12,7 \mathrm{MJ} \mathrm{m}^{-2} \mathrm{dia}^{-1}$, enquanto que para a Caatinga esta faixa foi de 8,9 a $12,3 \mathrm{MJ} \mathrm{m}^{-2} \mathrm{dia}^{-1}$. Com relação aos fluxos de calor latente $(\lambda \mathrm{E})$, sensível $(\mathrm{H})$ e no solo $(\mathrm{G})$ estas foram respectivamente de 8,5 a 10,2 e 0,5 a $1,9 \mathrm{MJ} \mathrm{m}^{-2} \mathrm{dia}^{-1} ;-0,2$ a 2,2 e 7,2 a $10,1 \mathrm{MJ} \mathrm{m}^{-2} \mathrm{dia}^{-1}$; e 0,3 a 0,5 e 0,2 a $0,4 \mathrm{MJ} \mathrm{m}^{-2} \mathrm{dia}^{-1}$. $\mathrm{H}$ foi algumas vezes negativo nas áreas bem irrigadas, significando advecção de calor horizontal de energia da vegetação natural mais seca. Como resultado da substituição das espécies Caatinga por culturas irrigadas a evapotranspiração (ET) incremental foi de até $4,0 \mathrm{~mm} \mathrm{dia}^{-1}$ no período mais seco do ano.

PALAVRAS-CHAVE: partição da energia, evapotranspiração, sensoriamento remoto

\section{ENERGY BALANCE DETERMINATION WITH LANDSAT 8 IMAGES IN THE NILO COELHO IRRIGATION SCHEME}

\begin{abstract}
In the Nilo Coelho irrigation scheme, located at the semi-arid region of Brazil, the natural vegetation, "Caatinga", has been replaced by irrigated agriculture, bringing importance of large-scale quantification of the energy balance components. Three Landsat 8 satellite images and four agro-meteorological stations were used together during periods of year 2014, under different thermohydrological conditions, for modelling these components in these mixed ecosystems. In irrigated crops, the net radiation $(\mathrm{Rn})$ average value varied from 9.1 to $12.7 \mathrm{MJ} \mathrm{m}^{-2}$ day $^{-1}$, while in "Caatinga" this range was from $8.9 \mathrm{a} 12.3 \mathrm{MJ} \mathrm{m}^{-2}$ day $^{-1}$. In relation to the latent $(\lambda \mathrm{E})$, sensible $(\mathrm{H})$ and soil $(\mathrm{G})$ heat fluxes, they were respectively from 8.5 to 10.2 and 0.5 a $1.9 \mathrm{MJ} \mathrm{m}^{-2}$ day $^{-1}$; -0.2 a 2.2 and 7.2 a $10.1 \mathrm{MJ} \mathrm{m}^{-2}$ day $^{-1}$; and 0,3 a 0,5 and 0.2 a $0.4 \mathrm{MJ} \mathrm{m}^{-2}$ day $^{-1} . \mathrm{H}$ was sometimes negative inside well irrigated areas, meaning horizontal heat advection from the drier natural vegetation. As a result of the replacement of the species from "Caatinga" by irrigated crops, the incremental evapotranspiration (ET) reached to $4.0 \mathrm{~mm}^{-1 a y}{ }^{-1}$ during the driest period of the year.
\end{abstract}

KEYWORDS: energy partition, evapotranspiration, remote sensing 


\section{INTRODUÇÃO}

A vegetação natural na região semiárida brasileira, a "Caatinga", tem sido rapidamente substituída pelas culturas irrigadas nos perímetros irrigados, sendo o Nilo Coelho, um dos mais importantes esquemas. Considerando-se os efeitos conjugados de mudanças de uso da terra e climáticos, torna-se muito relevante a aplicação de ferramentas para quantificação em larga escala dos componentes do balanço de energia nos ecossistemas mistos destes perímetros.

Dois algoritmos foram desenvolvidos e validados nas condições semiáridas brasileiras. O SAFER (Simple Algorithm For Evapotranspiration Retrieving) para a obtenção do fluxo de calor latente $(\lambda E)$ e o SUREAL (Surface Resistance Algorithm) para a estimativa da resistência da superfície aos fluxos hídricos $\left(\mathrm{r}_{\mathrm{s}}\right)$. Este último permite a classificação da vegetação em culturas agrícolas e Caatinga no semiárido brasileiro (Teixeira et al., 2004a, Teixeira et al., 2014b).

O objetivo da presente pesquisa foi a combinação das medições do Landsat 8 com dados de estações agrometeorológicas para a modelagem dos componentes do balanço de energia com aplicação destes algoritmos em diferentes condições termo hídricas no perímetro de irrigação Nilo Coelho.

\section{MATERIAL E MÉTODOS}

A Figura 1 apresenta a localização do Nilo Coelho no semiárido do Nordeste do Brasil e as estações agrometeorológicas utilizadas.

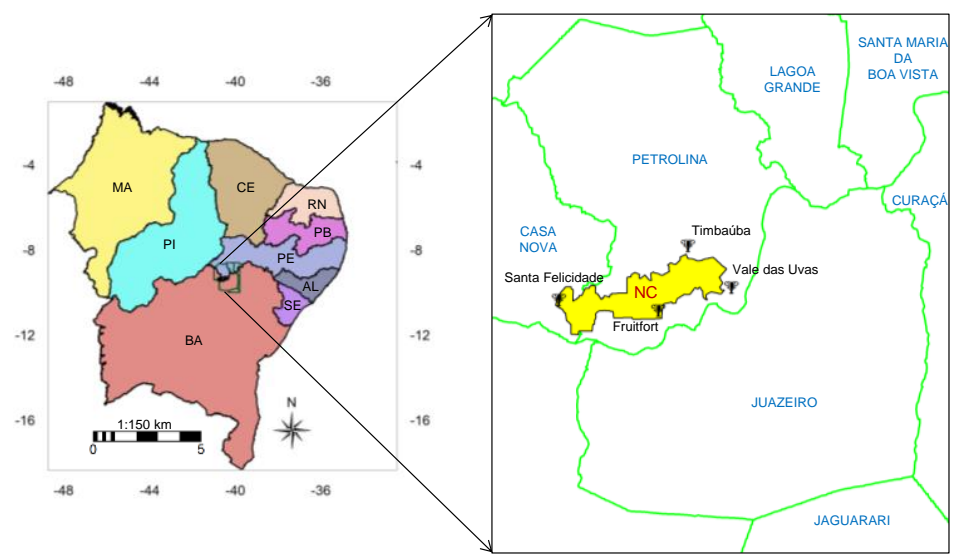

Figura 1. Localização do perímetro de irrigação Nilo Coelho no Nordeste do Brasil e estações agrometeorológicas utilizadas

As imagens Landsat 8 foram para os dias julianos - DJ 009 (09 de janeiro), 153 (02 de junho) e 265 (22 de setembro) no ano de 2014, representando condições dentro do período chuvoso, logo após as chuvas e no período seco da região semiárida brasileira. 
Para o cálculo do albedo da superfície $\left(\alpha_{0}\right)$ foram usadas as bandas de 1 a 7 , com resolução espacial de $30 \mathrm{~m}$, enquanto que para a temperatura da superfície $\left(\mathrm{T}_{0}\right)$ utilizaram-se as bandas termais 10 e 11, com resolução espacial de $100 \mathrm{~m}$, seguindo-se a metodologia descrita por Teixeira et al. (2014a) para os satélites Landsat 5 e 7 mas considerando-se os comprimentos de onda e constantes de conversão para o Landsat 8.

O SAFER foi usado para modelar os valores instantâneos da razão ET/ETo. A ET0 foi calculada pelo método de Penman-Monteith (Teixeira et al., 2014) nas estações agrometeorológicas da Figura 1, os valores interpolados e então a grade multiplicada pela razão $\mathrm{ET} \mathrm{ET}_{0}$ para obtenção da evapotranspiração atual (ET):

$$
\frac{\mathrm{ET}}{\mathrm{ET}_{0}}=\exp \left[\mathrm{a}_{\mathrm{s}}+\mathrm{b}_{\mathrm{s}}\left(\frac{\mathrm{T}_{0}}{\alpha_{0} \mathrm{NDVI}}\right)\right]
$$

onde $a_{s}$ e $b_{s}$ são os coeficientes de regressão, os quais foram 1,8 e $-0,008$, respectivamente, para as condições semiáridas do Brasil (Teixeira et al., 2014a).

Os valores diários de $\mathrm{R}_{\mathrm{n}}$ foram obtidos pela equação de Slob (Teixeira et al., 2014a,b):

$$
\mathrm{R}_{\mathrm{n}}=\left(1-\alpha_{0}\right) \mathrm{R}_{\mathrm{G}}-\mathrm{a}_{\mathrm{L}} \tau_{\mathrm{sw}}
$$

onde $a_{L}$ é um coeficiente de regressão que foi especializado de acordo com os valores de $T_{a}$ (Teixeira et al., 2014a,b).

O fluxo de calor no solo $(G)$ foi estimado através de sua relação com $R_{n}$ (Teixeira et al., 2014a,b):

$$
\frac{G}{R_{n}}=a_{g} \exp \left(b_{g} \alpha_{0}\right)
$$

onde $\mathrm{ag}_{\mathrm{g}}$ e $b_{\mathrm{g}}$ são coeficientes de regressão considerados 3,98 e -25,47.

Transformando-se a ET em unidades de energia estimou-se o fluxo de calor latente $(\lambda \mathrm{E})$ e o fluxo de calor sensível $(\mathrm{H})$ foi obtido como resíduo no balanço de energia:

$$
H=R_{n}-\lambda E-G
$$

Para classificação em culturas irrigadas e vegetação natural, o SUREAL foi aplicado, considerando limites nos valores de $\mathrm{r}_{\mathrm{s}}$ (Teixeira et al., 2014a).

$$
r_{s}=\exp \left[a_{r}\left(\frac{T_{0}}{\alpha_{0}}\right)(1-N D V I)+b_{r}\right]
$$


onde $a_{r}$ e $b_{r}$ são os coeficientes de regressão, considerados respectivamente 0,04 e 2,72.

\section{RESULTADOS E DISCUSSÃO}

A Figura 2 apresenta os valores diários da precipitação $(\mathrm{P})$ e evapotranspiração de referência $\left(\mathrm{ET}_{0}\right)$ obtidos na estação agrometeorológicas de Timbaúba (ver Figura 1) desde o início da estação chuvosa em 2013 ao início desta em 2014.

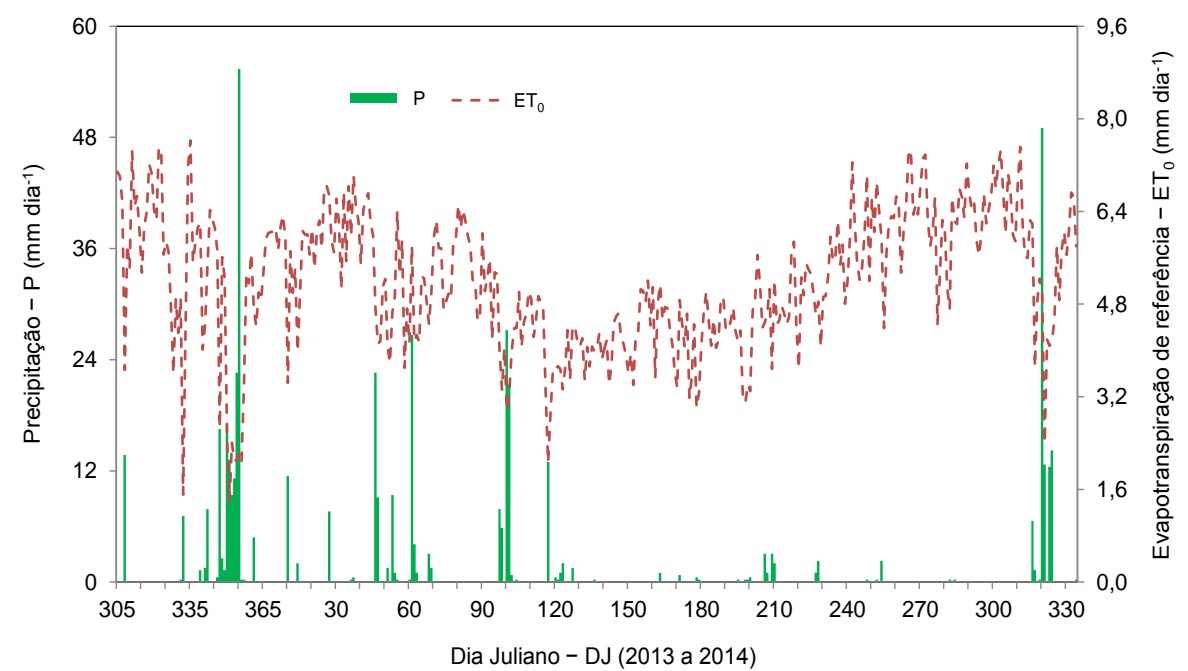

Figura 2. Precipitação $(\mathrm{P})$ e evapotranspiração de referência $\left(\mathrm{ET}_{0}\right)$ no período entre o início da estação chuvosa de 2013 ao início desta em 2014. DJ significa dia juliano

Considerando-se a imagem representativa do período chuvoso (09/01/2014), percebe-se que mesmo nestas condições, a última precipitação considerável aconteceu com antecedência de 19 dias. Em adição, um contínuo aumento nos valores de ET $_{0}$ é verificado, contribuindo com que as condições de umidade ficassem baixas. Para a imagem do período logo após as chuvas as chuvas (02/06/2014), a baixa demanda atmosférica contribuiu para que o solo ainda conservasse alguma umidade na zona das raízes das espécies da Caatinga. A imagem de 22/09/2014, por outro lado, além da ausência de precipitações, o contínuo incremento da $\mathrm{ET}_{0}$ contribuiu para um contraste maior entre a vegetação natural e as culturas irrigadas.

A Figura 3 mostra a distribuição espacial dos valores diários do saldo de radiação $\left(\mathrm{R}_{\mathrm{n}}\right)$ para as datas selecionadas no perímetro de irrigação Nilo Coelho.
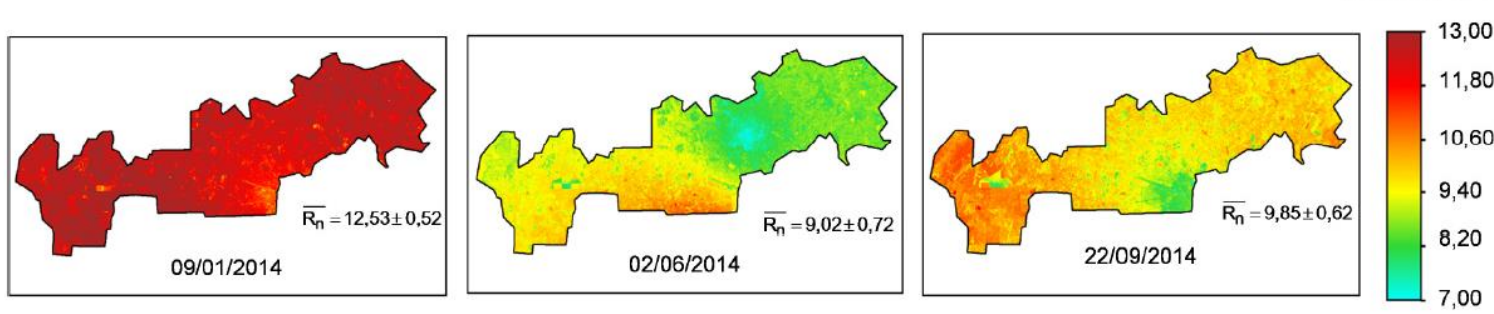

Figura 3. Distribuição espacial dos valores diários do saldo de radiação $\left(\mathrm{R}_{\mathrm{n}}\right)$ no perímetro de irrigação Nilo Coelho 
Em geral, há uma homogeneidade espacial nos valores de $\mathrm{R}_{\mathrm{n}}$, com baixos valores de desvio padrão (DP), independentemente das condições termo hídricas ao longo do ano de 2014. Não há uma distinção clara entre os pixels de $R_{n}$ das áreas irrigadas e de vegetação natural. Resultado similar foi encontrado por Teixeira et al. (2014b) em pivôs de milho no noroeste de São Paulo. A partição da energia foi investigada para os estes ecossistemas considerando-se os diferentes períodos do ano (Figura 4).
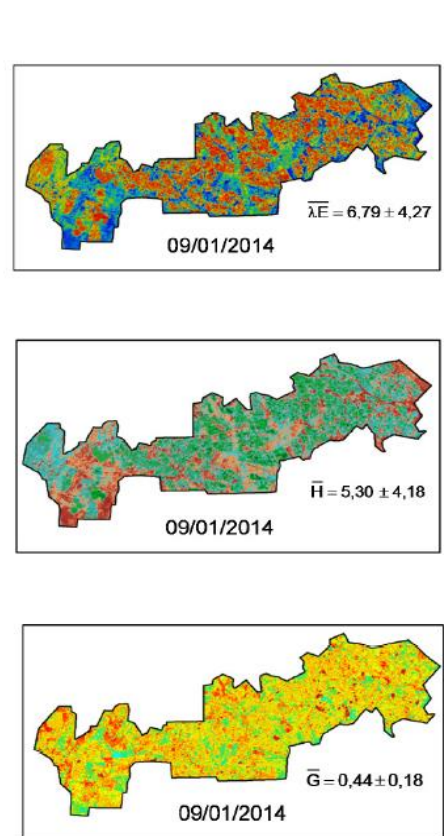

(a) Fluxo de calor latente $-\Lambda E$

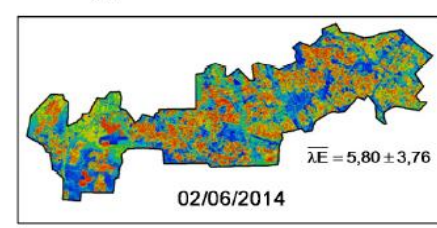

(b) Fluxo de calor sensivel - $\mathrm{H}$

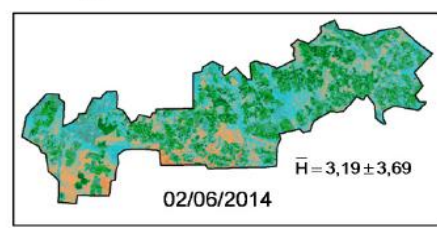

(c) Fluxo de calor no solo - G

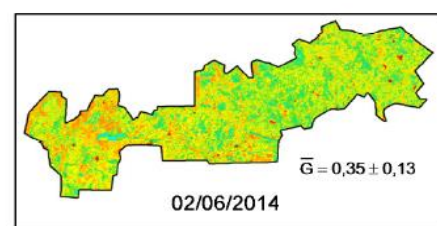

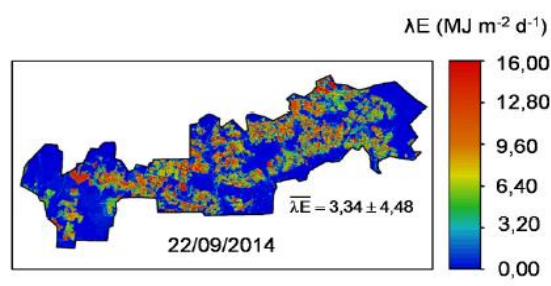

$\mathrm{H}\left(\mathrm{MJ} \mathrm{m}^{-2} \mathrm{~d}^{-1}\right)$

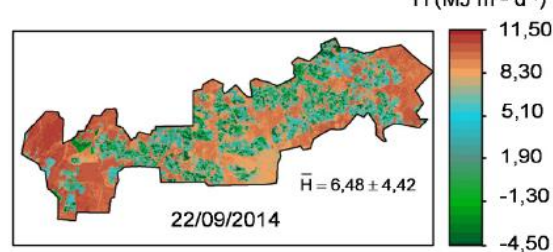

$\mathrm{G}\left(\mathrm{MJ} \mathrm{m}^{-2} \mathrm{~d}^{-1}\right)$

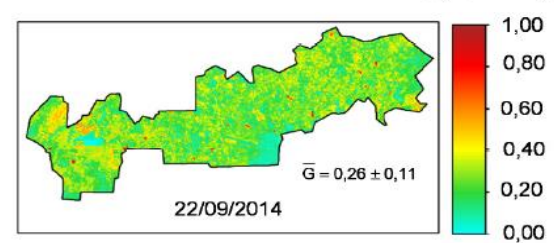

Figura 4. Distribuição espacial dos valores diários dos componentes do balanço de energia no perímetro de irrigação Nilo Coelho (a) fluxo de calor latente $(\lambda \mathrm{E})$; (b) fluxo de calor sensível (H); e (c) fluxo de calor no solo (G)

Claramente podem-se distinguir as áreas irrigadas pelos valores mais elevados de $\lambda \mathrm{E}$ (Figura 4a) e mais baixos de H (Figura 4b). Este último fluxo é alguma vezes negativo nas áreas bem irrigadas (coloração verde escura), significando advecção horizontal de energia das áreas mais secas vizinhas da Caatinga. Este fato foi também observado em pivôs de irrigação no noroeste de São Paulo (Teixeira et al., 2014b). Os valores diários de G foram próximos de zero, podendo de fato serem desprezados nesta escala de tempo.

A Tabela 1 mostra os valores médios e desvios padrões (DP) dos componentes do balanço de energia no distrito de irrigação Nilo Coelho. Os maiores valores de todos os componentes do balanço de energia ocorreram no período chuvoso representado pela imagem de DJ 009. Menores $\lambda E$ e H são no meio do ano, período logo após as chuvas e de menor demanda atmosférica (ver Figura 2). O período seco de maior advecção horizontal de calor é aquele representado pela imagem de DJ 265, quando $H$ é negativo e $\lambda E$ é acima de $R_{n}$ nas culturas irrigadas. Transformando-se os valores de $\lambda \mathrm{E}$ em ET, nas culturas irrigadas (CI) os 
valores médios variaram de acordo com a demanda atmosférica de 3,6 a 4,2 mm dia ${ }^{-1}$ respectivamente nas imagens de DJ 153 e 265, enquanto que a faixa de valores para Caatinga foi de 0,2 (DJ 265) a 0,8 (DJ 009) $\mathrm{mm} \mathrm{dia}^{-1}$, com ET incremental de até 4,0 $\mathrm{mm} \mathrm{dia}^{-1}$ no período mais seco (DJ 265).

Tabela 1. Médias diárias e desvios padrões do saldo de radiação $\left(\mathrm{R}_{\mathrm{n}}\right)$, fluxo de calor latente $(\lambda E)$, fluxo de calor sensível $(H)$ e fluxo de calor no solo $(G)$, no perímetro de irrigação Nilo Coelho

\begin{tabular}{lllllllll}
\hline \multirow{2}{*}{ DJ } & \multicolumn{2}{l}{$\mathrm{R}_{\mathrm{n}}\left(\mathrm{MJ} \mathrm{m}^{-2} \mathrm{dia}^{-1}\right)$} & \multicolumn{2}{l}{$\lambda \mathrm{E}\left(\mathrm{MJ} \mathrm{m}^{-2} \mathrm{dia}^{-1}\right)$} & \multicolumn{2}{l}{$\mathrm{H}\left(\mathrm{MJ} \mathrm{m}^{-2} \mathrm{dia}^{-1}\right)$} & \multicolumn{2}{l}{$\mathrm{G}\left(\mathrm{MJ} \mathrm{m}^{-2} \mathrm{dia}^{-1}\right)$} \\
\cline { 2 - 9 } & $\mathrm{CI}$ & $\mathrm{VN}$ & $\mathrm{CI}$ & $\mathrm{VN}$ & $\mathrm{CI}$ & $\mathrm{VN}$ & $\mathrm{CI}$ & $\mathrm{VN}$ \\
\hline 009 & $12.7 \pm 0.4$ & $12.3 \pm 0.6$ & $10.1 \pm 2.8$ & $1.9 \pm 1.0$ & $2.1 \pm 2.8$ & $10.1 \pm 1.0$ & $0.5 \pm 0.1$ & $0.4 \pm 0.2$ \\
153 & $9.1 \pm 0.7$ & $8.9 \pm 0.8$ & $8.8 \pm 2.5$ & $1.7 \pm 0.8$ & $0.2 \pm 2.5$ & $7.2 \pm 0.9$ & $0.4 \pm 0.1$ & $0.3 \pm 0.1$ \\
265 & $10.0 \pm 0.4$ & $9.8 \pm 0.7$ & $10.2 \pm 3.3$ & $0.5 \pm 0.7$ & $-0.2 \pm 3.2$ & $9.3 \pm 1.0$ & $0.3 \pm 0.1$ & $0.2 \pm 0.1$ \\
\hline Média & $10.6 \pm 0.5$ & $10.3 \pm 0.7$ & $9.7 \pm 2.9$ & $1.4 \pm 0.8$ & $0.7 \pm 2.8$ & $8.9 \pm 1.0$ & $0.4 \pm 0.1$ & $0.3 \pm 0.1$ \\
\hline
\end{tabular}

*CI - Culturas irrigadas; VN - Vegetação Natural; DJ - Dia Juliano.

\section{CONCLUSÕES}

O uso conjunto de imagens Landsat 8 e estações agrometeorológicas permitiram a quantificação em larga escala dos componentes do balanço de energia em diferentes condições termo hídricas do ano de 2014, considerando-se a mistura de agros-ecossistemas do Nilo Coelho, principal distrito de irrigação no semiárido brasileiro. Pôde-se concluir que o período chuvoso destaca-se como o de maiores valores de todos os componentes do balanço de energia, porém os maiores valores incrementais de evapotranspiração ocorrem no período mais seco do ano.

\section{REFERÊNCIAS}

TEIXEIRA, A.H. de C.; HERNANDEZ, F.B.T.; LOPES, H.L.; SCHERER-WARREN, M.; BASSOI, L.H. A Comparative Study of Techniques for Modeling the Spatiotemporal Distribution of Heat and Moisture Fluxes in Different Agroecosystems in Brazil. In: GEORGE G. PETROPOULOS. (Org.). Remote Sensing of Energy Fluxes and Soil Moisture Content. 1ed.Boca Raton, Florida: CRC Group, Taylor and Francis, 2014a, p. 169-191.

TEIXEIRA, A. H. de C.; HERNANDEZ, F. B. T. ; ANDRADE, R. G. ; LEIVAS, J. F. ; BOLFE, E. L. Energy balance with Landsat images in irrigated central pivots with corn crop in the São Paulo State, Brazil. Proceedings of SPIE - International Society for Optical Engineering, v. 9239, p. 923900-1-923900-10, 2014. 